\title{
Configuring and Enhancing Measurement Systems for
}

\section{Damage Identification}

\author{
Prakash Kripakaran ${ }^{1, a}$ and Ian. F. C. Smith ${ }^{1}$ \\ ${ }^{1}$ Applied Computing and Mechanics Laboratory (IMAC), Ecole Polytechnique
}

Fédérale de Lausanne (EPFL), Station 18, 1015 Lausanne, Switzerland

aCorresponding author, Email: prakash.kripakaran@epfl.ch, Phone: (+41) 21693 6372, Fax: (+41) 216934748

Abstract. Engineers often decide to measure structures upon signs of damage to determine its extent and its location. Measurement locations, sensor types and numbers of sensors are selected based on judgment and experience. Rational and systematic methods for evaluating structural performance can help make better decisions. This paper proposes strategies for supporting two measurement tasks related to structural health monitoring - (1) installing an initial measurement system and (2) enhancing measurement systems for subsequent measurements once data interpretation has occurred. The strategies are based on previous research into system identification using multiple models. A global optimization approach is used to design the initial measurement system. Then a greedy strategy is used to select measurement locations with maximum entropy among candidate model predictions. Two bridges are used to illustrate the proposed methodology. First, a railway truss bridge in Zangenberg, Germany is examined. For illustration purposes, the model space is reduced by assuming only a few types of possible damage in the truss bridge. The approach is then applied to the Schwandbach bridge in Switzerland, where a broad set of damage scenarios is evaluated. For the truss bridge, the approach correctly identifies the damage that represents the behaviour of the structure. For the Schwandbach bridge, the approach is able to significantly reduce the number of candidate models. Values of candidate model parameters are also useful for planning inspection and eventual repair.

Keywords: system identification; sensor placement; entropy; global search; damage identification; greedy strategy 


\section{Introduction}

Early detection of damage can prevent structural collapse and especially costly interventions by enabling preventive repair. To make appropriate decisions for maintenance, engineers need to know the type, the location and the extent of damage. Today many bridges and other structures are monitored using sophisticated measurement systems employing hundreds of sensors. For instance, the Stonecutters Bridge in Hong Kong with a main span of approximately $1 \mathrm{~km}$ has almost 1200 sensors [1]. Due to the lack of a systematic approach for the configuration of measurement systems, sensor types and locations are generally chosen based only upon engineering judgement. This strategy may not result in sensors that are placed at the most informative locations. Processing of large amounts of redundant data results in high data interpretation costs. Furthermore, in many cases, results from data interpretation are often inconclusive [2]. This paper evaluates systematic methods for measurement system design that maximize system performance and hence, support decisions with respect to the optimal number of sensors, sensor types and measurement locations.

Since the goal of measurement systems is to support data interpretation, measurement system performance must be related to its capacity to identify the state of the structure. The task of interpreting measurements falls into the broad area of system identification [3]. Research in system identification has focused on model updating techniques $[4,5]$ using both static [6-9] and dynamic loads [10-13]. Model updating methods estimate values of unknown parameters of a mathematical model of the structure on the basis of the assumption that the model that best fits measurements is the correct model. 
However, system identification is an inverse problem and errors are involved in measurement and modelling [9, 14-17]. This means that many damage scenarios [18] may explain the same measurements. Therefore a system identification approach that is based on a strategy of generation and iterative filtering of candidate models using measurement-interpretation cycles is more appropriate for structural management [19]. Sensor placement methods presented in this paper support such a strategy for system identification.

When structures show signs of damage such as increased deflection or large vibrations, engineers may decide to measure to increase their knowledge of the extent of damage and its location. Since detection of all possible damage requires that measurements are taken at all locations and in all directions, it is more practical to install an initial measurement system and enhance this system with additional sensors once damage is suspected and once measurement data has been interpreted. Consequently, this paper presents approaches for two measurement tasks- (1) design of initial measurement systems and (2) the iterative placement of sensors for model filtering and damage identification. Researchers who have studied the problem of sensor placement have focused on model updating using dynamic tests [20-23]. For system identification using multiple models, the initial measurement system must include sensors at locations that maximize the separation between damage scenarios.

Possible damage scenarios depend upon factors such as material, structural system, boundary conditions, loads and geographical location. For instance, one of the piers in a 
bridge may not be functioning as a support after a flood or an earthquake. Combinations of scenarios are also possible. Optimal sensor placement is a configuration that gives maximum separation between predictions of the effects of damage scenarios. Therefore, given the set of damage scenarios and a metric to compare sensor configurations on the basis of their ability to discriminate between damage scenarios, a global search can be used to find the optimal sensor configuration for the structure.

Since measurements from the initial measurement system are seldom sufficient for identifying the damage, additional sensors are placed iteratively to filter incorrect scenarios. In such cases, engineers need to know where to measure and what to measure. Robert-Nicoud et al. [24] proposed an iterative greedy algorithm that places sensors at locations that give maximum separation between predictions of candidate models. Entropy among candidate model predictions was used as a criterion for selecting subsequent measurement locations. If measurements from potential sensor locations discriminate efficiently between the damage scenarios, the strategy provides measurement locations that enable engineers to converge to the correct model.

In this paper, two optimization-based methods are presented for common measurement tasks in structural identification. A global search strategy is proposed for designing initial measurement systems. A greedy strategy is proposed for finding subsequent measurement locations for damage identification. Two case studies illustrate these methods. A truss railway bridge, derived from the specifications of the Zangenberg railway bridge in Germany, is the first case. The second case is the Schwandbach bridge 
in Switzerland. In both cases, damage scenarios are generated through varying characteristics such as those related to the structural system and the material.

\section{Multiple-Model System Identification}

The multiple model strategy presented in this paper is an iterative process that suggests measurement locations for identification and then uses information from identification to improve the measurement system. This strategy $[19,25]$ explicitly addresses the two main challenges of conventional model updating - effects of compensating uncertainties and the presence of multiple solutions, by

- sampling combinations of modelling assumptions and

- evaluating several candidate models such that model predictions are below threshold values defined according to measurement and modelling uncertainties

A flowchart of such a system identification process is shown in Figure 1. This flowchart is derived from previous research performed at EPFL. At the beginning, modelling assumptions and damage scenarios are provided by engineers based on their knowledge of structural behaviour and engineering judgement. Modelling assumptions are related to uncertainties that affect structural behaviour such as boundary conditions, material properties and rigidity of connections. These assumptions result in sets of numerical parameters involving quantities such as elastic modulus, moment of inertia, support stiffness and connection stiffness. Damage scenarios influence aspects of the structure such as its geometry, structural system and environment. Damage scenarios are also parameterized as described later in the paper. Each set of values for model parameters corresponds to a model of the structure. Predictions from models that represent several modelling assumptions and damage scenarios are used to design an initial measurement 
system. Later, modelling assumptions are combined with measurements to identify sets of candidate models that explain measured behaviour. Measurements are compared with model predictions in a stochastic search to generate the sets of candidate models.

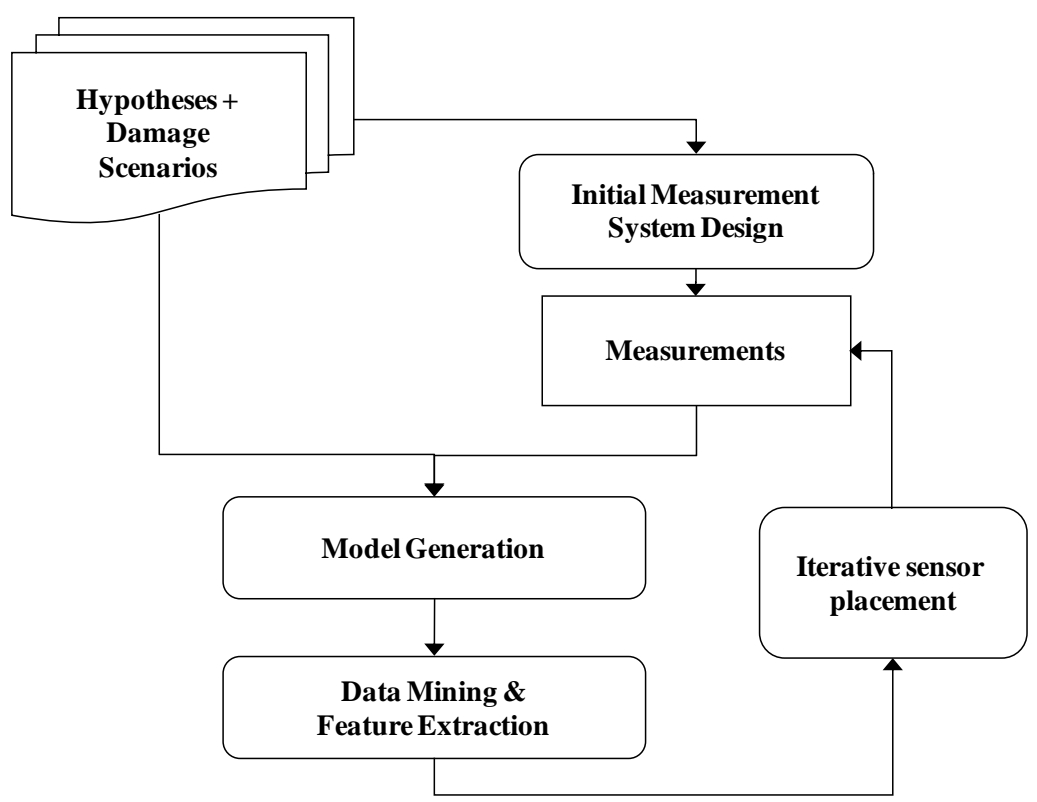

Figure 1: Flowchart of tasks in multiple-model system identification

Robert-Nicoud et al. [25] defined the objective function $E$ for stochastic search as follows.

$$
E=\left\{\begin{array}{l}
\varepsilon, \text { if } \varepsilon>\tau \\
0, \text { if } \varepsilon \leq \tau
\end{array} \quad \text { and } \varepsilon=\sqrt{\sum\left(m_{i}-p_{i}\right)^{2}}\right.
$$

$\varepsilon$ is the residual and is calculated as the difference between predictions $p_{i}$ and measurements $m_{i} . \tau$ is a threshold value evaluated from measurement and modelling errors in the identification process. The set of models for which $E=0$ is the set of 
candidate models for the structure. In this study, the objective function has been modified as follows.

$$
\begin{gathered}
f_{i}=\left\{\begin{array}{l}
0, \text { if } \varepsilon_{i}<\tau_{i} \\
\varepsilon_{i}^{2}, \text { if } \varepsilon_{i}>\tau_{i}
\end{array} \text { and } \varepsilon_{i}=\left|m_{i}-p_{i}\right|\right. \\
E=\sqrt{\sum_{1}^{n} \frac{f_{i}}{n}}
\end{gathered}
$$

$\varepsilon_{i}$ is the difference between model prediction and measurement at location $i . \tau_{i}$ is a threshold value for location $i . \tau_{i}$ is determined according to the sensor accuracy and the prediction uncertainty at location $i$. Candidate models are those for which $E=0$. Equations (2) and (3) ensure that stochastic search finds sets of candidate models such that each candidate model has a prediction that is below a certain threshold value at each measurement location. This objective function improves upon Equation (1) by allowing the definition of a threshold value for each sensor location. Figure 2 graphically illustrates the selection of candidate models. The residual $\left(\varepsilon_{1}\right)$ for each model is plotted with respect to the value for a model parameter. The threshold value $\left(\tau_{1}\right)$ for candidate models is shown by a dashed line. All models with residuals below the dashed line are selected as candidate models. 


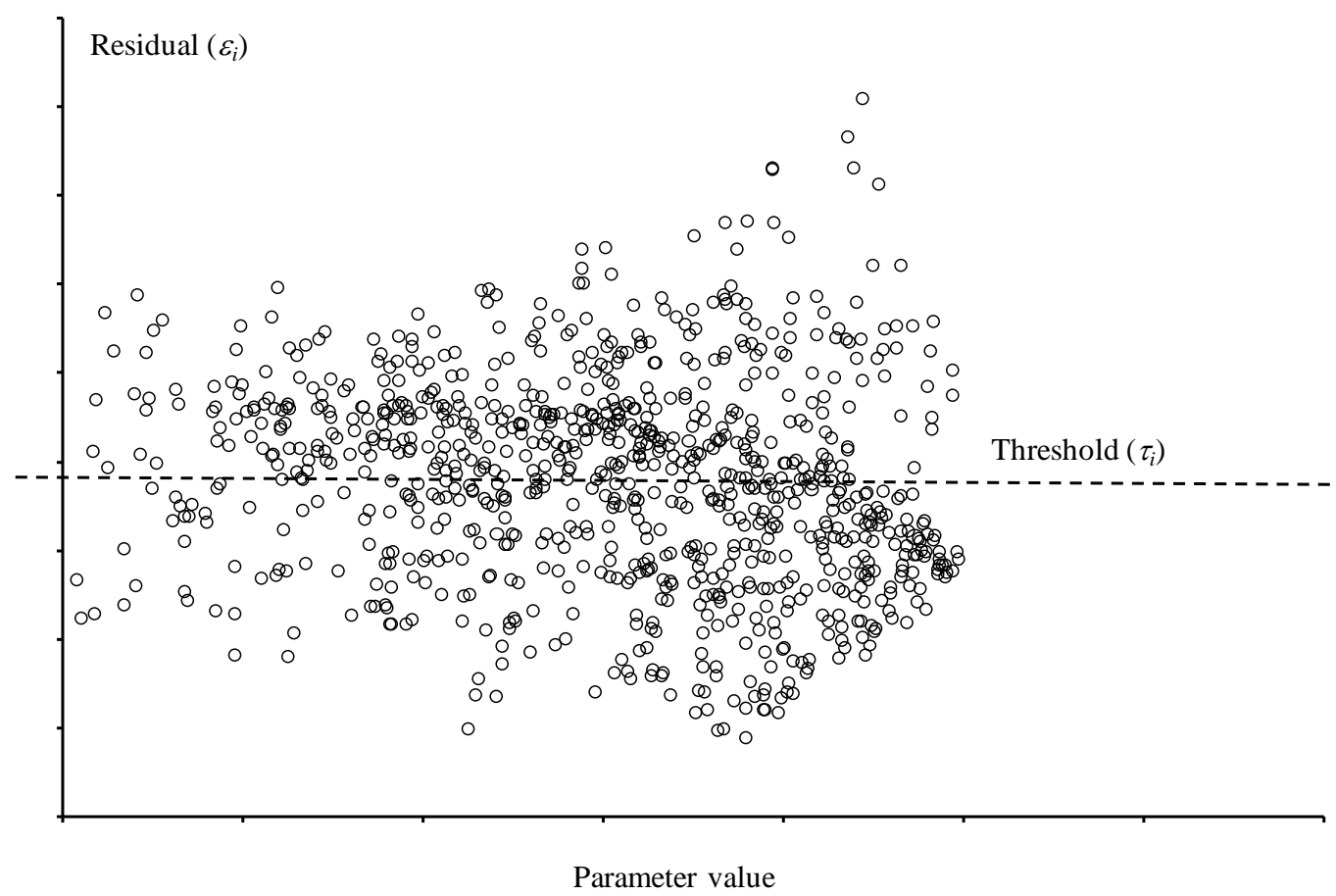

Figure 2: The residual between model predictions and measurements is plotted for a given parameter value. Each circle is the prediction of a model. The threshold value $(\tau)$ is shown by a dashed line.

Complex structures have large numbers of candidate models. These are examined by a data mining and feature extraction module (Figure 1). Data mining techniques are used to cluster similar models and extract relationships between models. Decisions on subsequent measurements are taken based on entropy calculations of predictions of representative models from different model clusters.

\section{Optimal Sensor Placement}

Sensor-data driven decision support systems can be the basis of proactive management of structural facilities [26]. For effective decision support, it is essential that sensor systems are configured to measure responses at locations such that the measured data can be meaningfully interpreted. The sensor placement methodology proposed in this 
paper requires two key components - (1) an approach for generating damage scenarios and (2) a method for evaluating the performance of sensor configurations.

\section{Generation of Damage Scenarios}

Examples of damage scenarios are abutment settlement and stiffness loss in a truss element. These scenarios depend upon structural factors such as material, geometry, structural system characteristics and geographical location. Each of these scenarios is represented by one or more parameters which have values between ranges specified by engineers. For example, damage in a beam may be modeled as the percentage reduction in the flexural stiffness and it may vary between $50 \%$ and $100 \%$ reduction. Scenarios can later be combined using model composition [19] to generate additional damage scenarios. For example, the following two scenarios - damage in a beam and settlement of an abutment, may be combined to generate another damage scenario. Since generating all possible scenarios is combinatorial, a population is randomly generated using assumptions that are declared by the engineer. Each scenario is evaluated by finite element analysis. Its predictions $p_{i}$ at all possible sensor locations are computed and stored in a set $M_{0}$. A sampling error may be introduced when insufficient numbers of models are sampled. Thus, there are $N$ sets of predictions $p$ in $M_{0}$.

\section{Sensor System Performance Evaluation}

The goal of measurement-system configuration is to place sensors at locations that offer maximum separation between model predictions. In this paper, we specify the performance of a sensor configuration in terms of the maximum number of models that cannot be discriminated using measurements from the configuration. A model from this set of non-separable models is the worst-case scenario for the configuration. Given a 
sensor configuration with $s$ number of sensors, the worst-case scenario is evaluated as follows.

- At each location $i$ where a sensor is placed, models are grouped into sets according to the values of their predictions at location $i$. These sets may be evaluated by plotting a histogram for the model predictions in $M_{0}$. The number of intervals $I$ for the histogram is fixed according to the accuracy of the sensors and the model predictions in $M_{0}$. An example of a histogram is shown in Figure 3. Each bar in the histogram represents the number of models whose predictions lie within its interval.

- Define model sets such that each set contains only those models whose predictions lie within the same interval. There are $I$ sets for a histogram at location $i$ and these are grouped into a super-set $B_{i}$. Thus, $B_{1}, B_{2} \ldots B_{s}$ are the sets obtained by evaluating histograms at sensor locations 1 to $s$.

- The maximum number of non-identifiable models $U_{\max }$ is given as the maximum possible size of the set B given by

$$
B=\left\{b_{1} \cap b_{2} \cap b_{3} \ldots \cap b_{s}\right\}
$$

$b_{i}$ is an element of set $B_{i}$. Thus the objective of the optimal sensor placement problem is to minimize the value of $U_{\max }$. 




Figure 3: Histogram of model predictions

\section{Global Search}

PGSL [27] is a direct search algorithm that employs global sampling to find the minimum of a user defined objective function. Gradient calculations are not needed and no special characteristics of the objective functions (such as convexity) are required. PGSL has been successfully applied to optimization problems in design configuration, structural control [28] and system identification [19].

PGSL is used in this study for designing initial measurement systems for structures. The purpose is to evaluate optimal sensor configurations given the potential sensor locations and the required total number of sensors. Primary input to PGSL is the number of variables and the range of acceptable values for each variable. For the sensor placement problem, the number of decision variables is equal to the number of potential sensor locations. Minimizing $U_{\max }$ is the objective. 
The stochastic sampling nature of PGSL means that it operates best on continuous variables. However, the variables for the sensor placement problem are binary decision variables representing the presence or absence of a sensor at each sensor location. To overcome this problem, each variable is modeled as continuous and varying between 0 and 1 in PGSL. Consider the case when PGSL is used to find the optimal sensor locations for number of sensors equal to $I$. Then each solution generated by PGSL is interpreted as having sensors only at those locations corresponding to variables with the $I$ largest values.

To arrive at the best number of sensors, optimal sensor configurations are evaluated for different specified numbers of sensors. Engineers can then compare the costs of having additional sensors against the gain in overall performance. Similarly performance improvement upon adding sensors is evaluated by computing the difference between $U_{\max , i+1}$ and $U_{\max , i .}$. This difference is the reduction in the number of non-identifiable models between sensor configurations with $i$ and $(i+1)$ sensors respectively. A very small difference indicates that only limited information is acquired by having an additional sensor. Ideally, it is preferable to have sufficient sensors to reduce the number of non-identifiable models $U_{\max }$ to 1 . However, such a situation is seldom feasible due to limitations in cost and sensor accuracy.

\section{Iterative Model Filtering}

This section introduces the greedy strategy for finding subsequent measurement locations during damage identification. Upon observing anomalous behavior in a bridge, systematic approaches help find the best locations to measure next so that the cause 
(damage) of such behavior is identified as soon as possible. A set of candidate damage scenarios is generated that match the measurements from existing sensors on the bridge. Sufficient numbers of damage scenarios are sampled to ensure that a scenario equivalent to the real damage location is present in this set. Subsequent measurement locations can be found on the basis of the entropy $[29,30]$ of candidate predictions at various possible measurement locations. Locations with high entropy values are likely to eliminate the maximum number of models from the candidate set. Therefore, the method finds subsequent measurement locations such that the likelihood of rapidly arriving at the damage locations is high.

Shannon's entropy function [29] is a mathematical representation for the uncertainty in a set. This expression comes from the field of information theory and it formulates the disorder within a set. For a random variable $X$, the entropy $H(X)$ is given by the following equation.

$$
H_{s}=-\sum_{j} P_{j} \cdot \log _{2} P_{j}
$$

$P_{j}$ are the probabilities of the $|X|$ different possible values of $X$. For practical purposes, $0 \cdot \log (0)$ is taken to be 0 . When a variable takes $|X|$ discrete values, the entropy is a maximum when all values have the same probability $\log _{2}(X)$. Thus entropy is a measure of homogeneity in a distribution.

The entropy for a given sensor location is calculated from the histogram of model predictions (Figure 3). The probability $P_{j}$ of an interval is the ratio of the number of models in the interval to the total number of models. At the best measurement locations, model predictions should have maximum variation. $P_{j}$ is the probability of the $j$ th 
interval in the prediction distribution at location $s . P_{j}$ is calculated as the ratio between the number of models that have predictions within the $j$ th interval and the total number of models. If model sets have high values of entropy, more candidate models can be filtered. The location at which $H_{s}$ is a maximum is chosen for subsequent measurement. If the entropy among predictions is insignificant (close to zero), then additional measurement locations or sensor types may be required for further eliminating models. Model filtering is stopped when only a single candidate model remains (model identification). In the following sections, we illustrate these two algorithms - global search and entropy-based sensor placement, for measurement system design using two case studies.

\section{Case Study One: Zangenberg Bridge}

A structure inspired from the railway bridge in Zangenberg, Germany is considered as a case study. This case study illustrates (1) the design of an initial measurement system for the bridge and (2) damage identification using iterative placement-measurementinterpretation cycles. The bridge is composed of two parallel trusses. A diagram of a single truss is shown in Figure 4. Each truss has 77 members and spans a length of 80 m. Loads from the wagons and locomotives are transmitted to the trusses by transverse beams. The two parallel trusses also have wind bracings between the top and bottom chords. The properties of the truss members are given in Table 1. The truss members are made of steel having an elastic modulus of $200 \mathrm{GPa}$ and a density of $7870 \mathrm{~kg} / \mathrm{m}^{3}$. The truss is fixed at one end of its span and the other end is supported on a roller. Truss members can suffer damage due to fatigue loading and corrosion. In this example, damage is considered to result in a loss in member stiffness. 


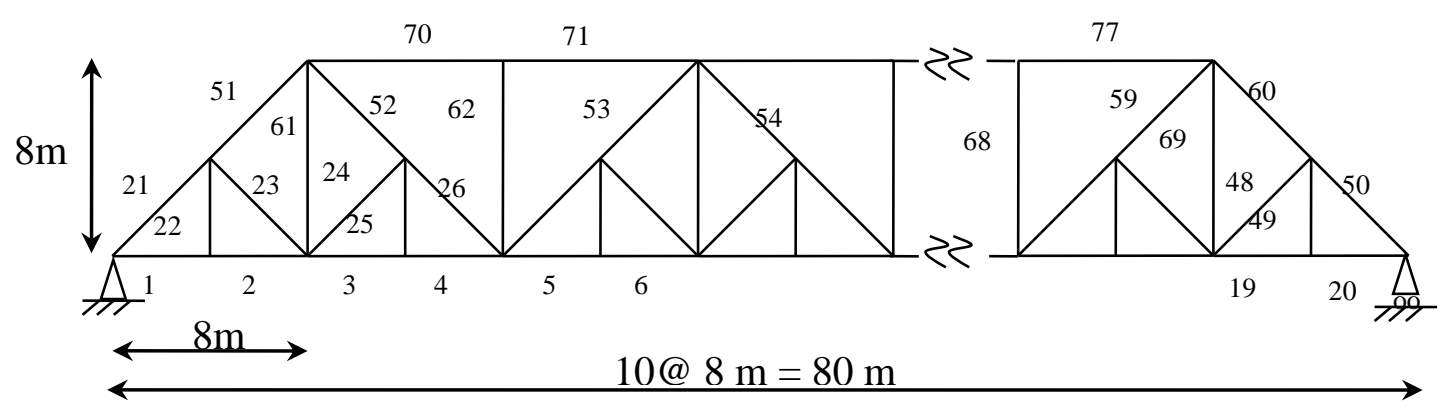

Figure 4: A truss inspired from the railway bridge in Zangenberg, Germany

Since measurements from a real damage scenario are unavailable, simulations using finite element models are used. The responses (axial strains) of the finite element model in the different members are taken as the measurements from the field. Let us assume the following scenario. Engineers notice a significant increase in mid-span deflection and decide to test the bridge for damage identification. A load equivalent to a railway locomotive is used for a static load test. This is transferred to the truss in the form of four concentrated loads at four nodes starting from the $9^{\text {th }}$ node from the left end of the bottom chord in Figure 4. Each concentrated load has a value of $250 \mathrm{kN}$.

For illustration purposes, this paper simplifies the example to a case where the candidate model space is enumerable. One truss of the bridge is modelled. An initial measurement system is sought to detect damage scenarios in which a single member of the truss is assumed to be damaged. Subsequent measurement locations are then sought to filter incorrect models and identify the correct damage scenario. The damage is assumed to result in a 50\% loss of axial stiffness. Strain gauges are the measurement devices chosen for this study as they are the most commonly used sensors for steel structures. They are taken to have a precision of 1 microstrain. For simplicity, other errors are assumed to be zero. In this case study, damage is simulated in member 8 . 
Table 1: Properties of truss members in Zangenberg bridge

\begin{tabular}{llll}
\hline \multicolumn{1}{c}{ Member type } & \multicolumn{1}{c}{ Area $\left(\mathrm{cm}^{2}\right)$} & \multicolumn{1}{c}{$\mathrm{I}_{\mathrm{x}}\left(\mathrm{cm}^{4}\right)$} & $\mathrm{I}_{\mathrm{y}}\left(\mathrm{cm}^{4}\right)$ \\
\hline Top chord & 0.0515 & $2.267 \times 10^{-3}$ & $2.586 \times 10^{-3}$ \\
Bottom chord & 0.0303 & $1.467 \times 10^{-3}$ & $1.458 \times 10^{-3}$ \\
Vertical & 0.0219 & $1.215 \times 10^{-3}$ & $4.245 \times 10^{-5}$ \\
Diagonal & 0.0369 & $9.704 \times 10^{-4}$ & $4.164 \times 10^{-3}$ \\
Small diagonal & 0.0219 & $1.215 \times 10^{-3}$ & $4.245 \times 10^{-5}$ \\
\hline
\end{tabular}

Several modelling assumptions have been made during design of this truss. The connections in the truss were assumed to be hinged for design purposes. However, the rivet-plated connections which are used in this bridge are known to transfer moments. Moreover, studies on connection behaviour have shown that truss joints typically behave in a semi-rigid manner. There are also uncertainties in the behaviour of the truss members. They could be modelled either as trusses elements which only take axial forces or as beam elements. These modelling assumptions can be explicitly considered during multiple-model system identification. For this example, two assumptions are explored - (1) truss elements with hinged joints and (2) beam elements with rigid joints.

\section{Results}

Even though this problem has been simplified by assuming that damage occurs in only one member and by modelling only a few assumptions, many combinations of damage scenarios and assumptions match the measurements. A strategy of model filtering through iterative measurement-interpretation cycles is used to find the correct model. As a first step, all models in the model space are evaluated. Since there are two modelling assumptions and each model can have only one damaged member, there are $154(77 \times 2)$ damage scenarios. All models are generated by exhaustive search. For each model, the 
strain prediction in each member is evaluated using finite element analyses. Global search is used to find the optimal sensor configuration for the truss bridge.

There are 77 potential sensor locations corresponding to the total number of members in the truss. Symmetry in sensor positions is enforced during optimization. Figure 5 shows the results from optimization. The number of non-identifiable models initially reduces rapidly with increasing number of sensors. However, it later tapers off and remains constant at 4 for more than 12 sensors. Thus, the gain in performance diminishes with increasing number of sensors. As the performance improvement is only marginal for a number of sensors greater than 8 , an initial measurement system with 8 sensors may be installed on the bridge. The sensors are installed on members 5, 16, 21, 28, 43, 50, 54 and 57.

Measuring at these eight locations is not sufficient to identify the correct damage scenario. After using these measurements to filter models from the set of damage scenarios, many damage scenarios still remain. Filtering is the process of eliminating models for which $E$ as calculated using Equation (3) is unequal to 0. The initial measurements are able to filter 135 models. The size of the new set of candidate models is 19 . Thus, 19 damage scenarios are possible given these 8 measurements. Further measurements are required to identify the correct damage scenario. The entropies of the predictions of the reduced set of models at the various possible measurement locations are computed using Equation (4). The maximum entropy among the axial strain predictions is at member 30. In the first iteration, a strain sensor is placed on member 
30. The new measurement is used to again filter models using Equation (3). Measuring at this location filters 15 models. 4 damage scenarios still remain that are equally likely to explain the behaviour of the bridge. Again entropies among candidate model predictions are computed at the remaining potential sensor locations. The maximum entropy is found at member 7. In the second iteration, a strain sensor is placed on member 7 . The new measurement filters 3 models. The remaining model exactly matches the damage simulated for the structure. A notable result is that the method did not place any sensor on the damaged member.

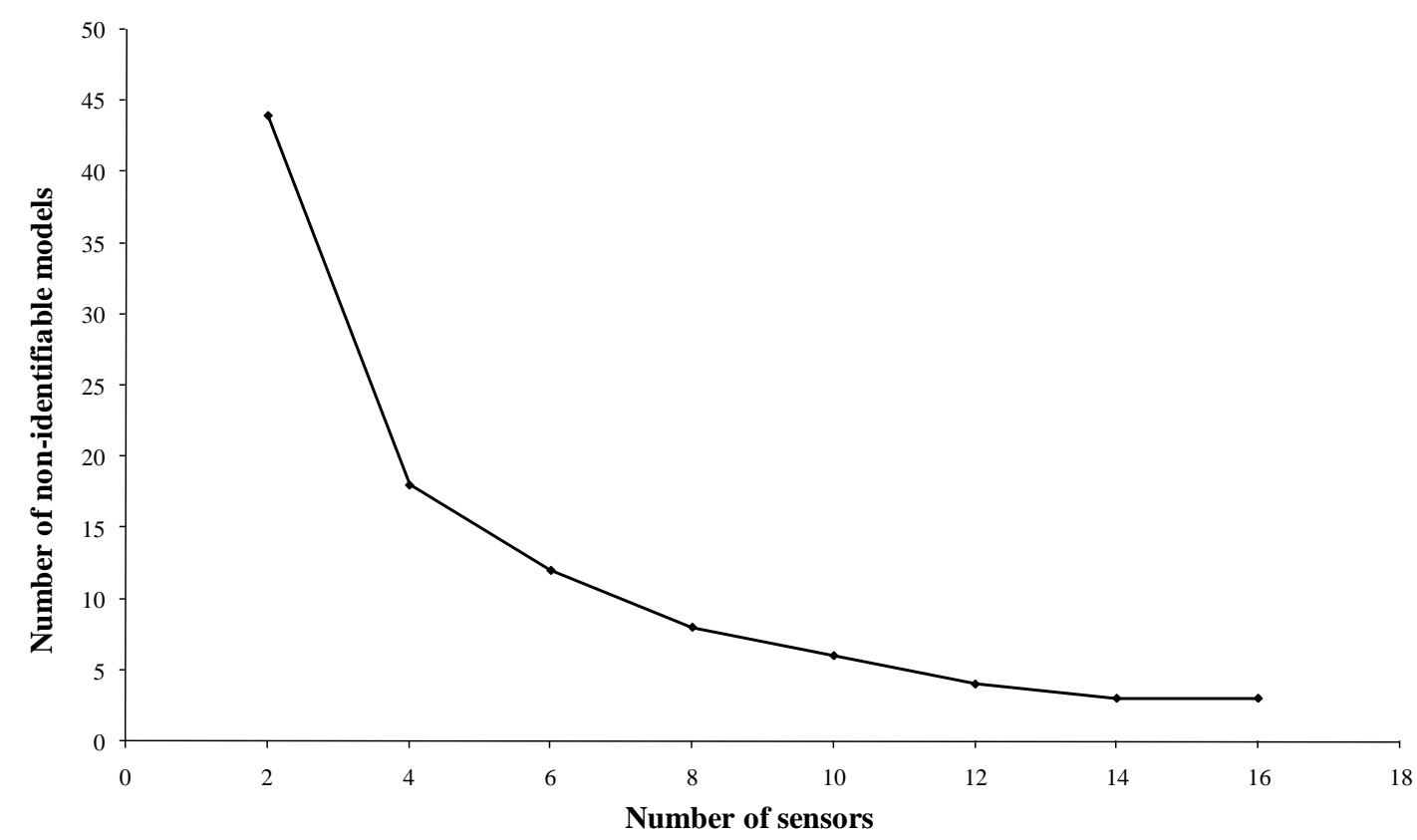

Figure 5: Plot showing the variation of maximum number of non-identifiable models with number of sensors for the bridge in Figure 4

In practice, complete enumeration of candidate model space as illustrated in this example is rarely possible. In such cases, a stochastic search [27] that employs the objective function given in Equation (3) can be used to find the candidate models. To illustrate such a case, the Schwandbach bridge is examined next as Case Study Two. 


\section{Case Study Two: Schwandbach Bridge}

A bridge that was designed by Maillart in 1933 (Figure 6) is used to illustrate the sensor configuration methodology. Still standing today, the Schwandbach bridge is an early example of a deck-stiffened open-spandrel arch and has been named by Billington [31] "to be one of the two or three most beautiful concrete bridges ever built". The elliptic horizontal ground-plan curve that is supported by a vertical curved thin-walled arch is also an example of daring structural engineering that has inspired engineers for over seventy years.

\section{ELEVATION}
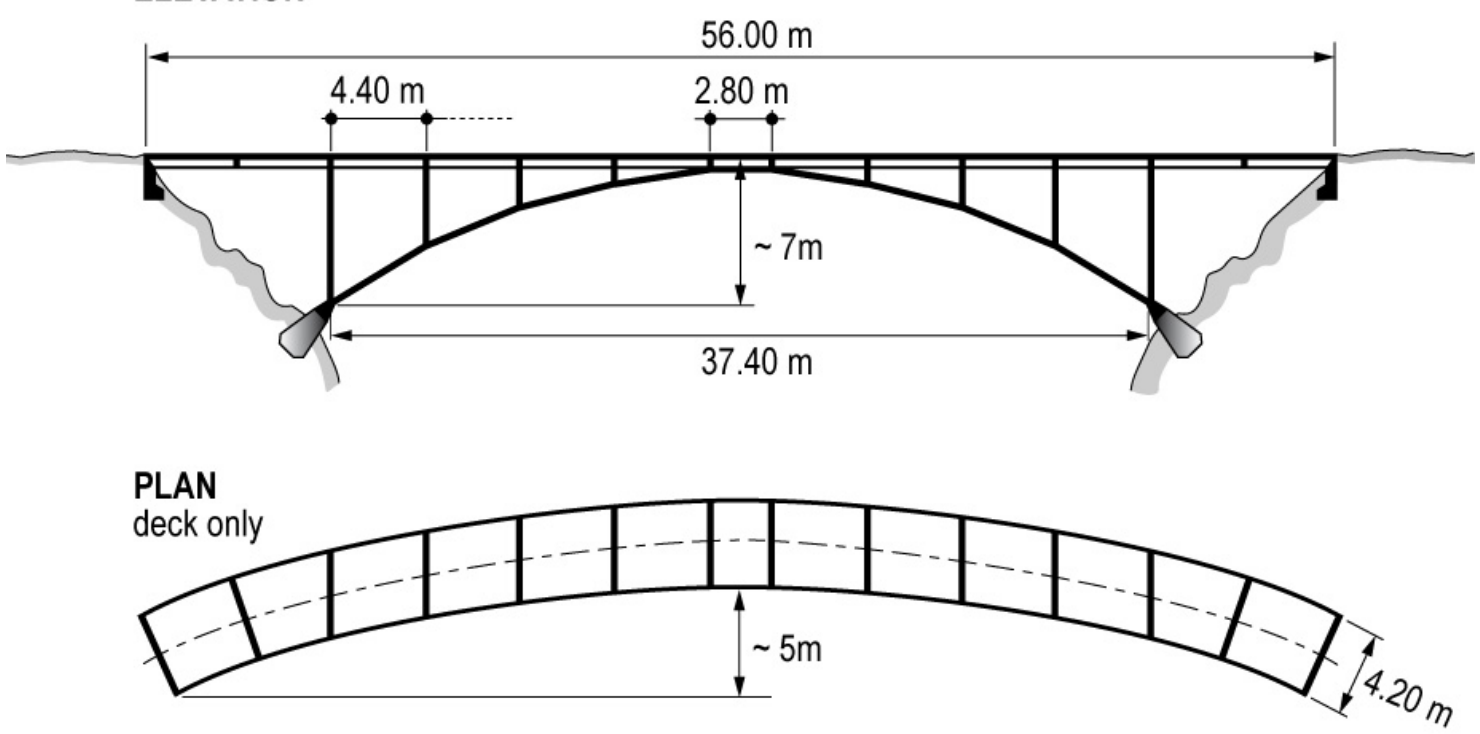

Figure 6: Plan and elevation of Schwandbach bridge in Switzerland

The possible sensor locations on the bridge are shown in Figure 7 using node identification numbers. Since this is a bridge with a short span of approximately $50 \mathrm{~m}$, the deflections of the bridge are relatively small. Displacement sensors that can measure with an accuracy of up to $1 \mathrm{~mm}$ are found to be insufficient for system identification. However, inclinometers with fairly high accuracy (approximately 1 microradian) are available and this resolution is acceptable for such system identification. In the 
following section, damage scenarios that determine the location of the inclinometers on the bridge are explained.

\section{Damage scenarios}

Incorporation of damage scenarios in the sensor placement methodology enables selection of measurement locations that maximize the likelihood of identifying future damage. The damage scenarios that are used in this case study are listed in Table 2 . The scenarios are derived from a previous experimental study on the Z24 bridge in Switzerland by Maeck et al.[32]. Maeck et al. [32] created a similar damage in the structure and made measurements under dynamic and static loads. Table 2 also lists the degree of damage to be simulated under each scenario.

Table 2: Damage scenarios considered and possible causes

\begin{tabular}{lll}
\hline Damage & Causes & Damage limits \\
\hline Arch abutment settlement & Settlement of subsoil, erosion & $0-30 \mathrm{~cm}$ \\
Arch abutment tilt &,, & $0-2^{\circ}$ \\
Deck support settlement &, & $0-30 \mathrm{~cm}$ \\
Cracks on inner girder & $\begin{array}{l}\text { Overload, settlement of subsoil, } \\
\text { erosion }\end{array}$ &, $50 \%$ reduction \\
Cracks on outer girder &,, &,, \\
Spalling of inner girder & $\begin{array}{l}\text { Frost thawing cycles, } \\
\text { temperature shock while }\end{array}$ & \\
Spalling of outer girder & applying de-icing salt &,, \\
\hline
\end{tabular}

In this study, the bridge is modelled as a finite element model in ANSYS. Damage scenarios are simulated by changing the parameters within the finite element model. Arch abutment settlement and tilt as well the deck support settlement are close to the ends of the structure. Simulations revealed that the girder elements in the span between the ends and the first vertical slab experience the maximum stress and hence, cracks and spalling are also assumed to occur close to the ends. 
Candidate models are generated by stochastic sampling in a model space that consists of all combinations of the damage scenarios listed in Table 2. The damage that causes the real behaviour of the bridge as measured by the sensors is a settlement of $5 \mathrm{~cm}$ at the left arch abutment.

\section{Results}

A set containing 5000 damage models of Schwandbach bridge is created in order to represent the space of possible models. The number of possible sensor locations is 20 (see Figure 7 for details). The size of the solution space is $2^{20}$ since a sensor may or may not be present at a given location. This space is sufficiently large to illustrate key aspects of how this methodology scales up to large initial solution spaces.

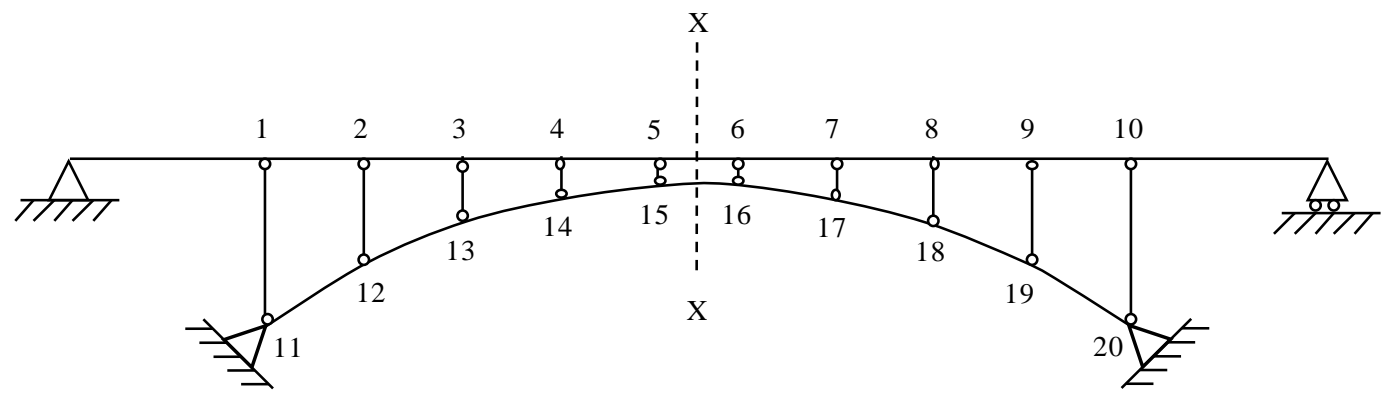

Figure 7: Potential sensor locations on Schwandbach bridge

As in the previous case study, engineers decide to measure the structure for damage due to observed behavioural change. The purpose of the measurements is to determine the location and extent of damage. Since the damage scenarios listed in Table 2 have continuous parameters, simulation of all possible combinations is impossible. Therefore, 5000 damage scenarios are generated through sampling. The interval size for the histograms depends on the sensor precision, i.e., 1 microradian for inclinometers and the modelling error (4 microradians). Therefore the interval size in this case study is 5 
microradians. Global search is used to identify optimal sensor configurations for increasing numbers of sensors. Schwandbach bridge is nearly symmetrical with respect to a center line (depicted by line $\mathrm{X}-\mathrm{X}$ in Figure 7) and the damage scenarios considered in this study are also symmetrical. Therefore, the sensor placement algorithm explicitly imposes symmetry. Results from global search are plotted in Figure 8.

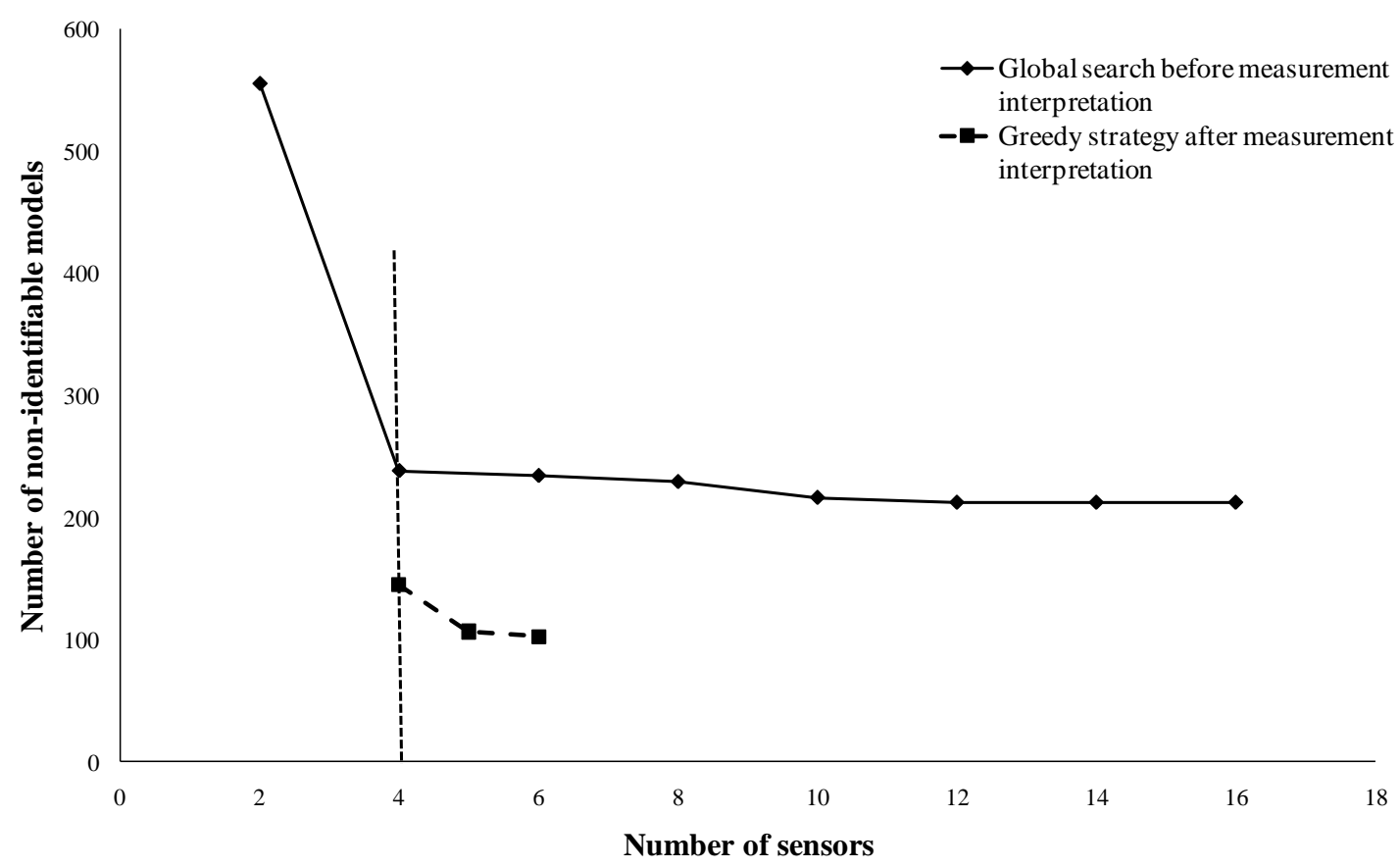

Figure 8: The variation using global search of maximum number of non-identifiable models with number of sensors for the Schwandbach bridge, and model filtering using a greedy strategy when starting with measurements from an initial measurement system having 4 sensors (vertical dashed line) are shown.

For values of number of sensors greater than 12 , there is no decrease in the number of non-identifiable models. This means that placing more than 12 sensors will not benefit the damage identification process. In fact, the gain in performance decreases rapidly after placing 4 sensors on the bridge. Therefore, an initial measurement system with 4 sensors is installed on the structure. The inclinometers are placed at locations 1, 2, 9 and 10 (see Figure 7). The sensor positions suggested are close to the elements that have been modified to represent damage. 
From the set of 5000 damage scenarios, measurements from these 4 locations eliminate 4855 scenarios. Therefore, there are still 145 scenarios that are equally likely to explain the behaviour of the bridge. This set of 145 scenarios is the initial set of candidate scenarios for the greedy strategy and is indicated by the first data point of the greedy strategy curve in Figure 8. The global search curve in Figure 8 is an upper-bound to the performance of the greedy strategy since it evaluates the worst-case performance of measurement systems with a specified number of sensors. The greedy strategy is used after filtering models using a set of measurements. During each iteration the greedy strategy identifies the best location for the next sensor based on entropy among candidate model predictions. In the first iteration, the algorithm finds the maximum entropy at sensor location 19. Measuring at location 19 eliminates 38 scenarios. There are 107 candidate damage scenarios after filtering models. In the next step, the greedy strategy evaluates maximum entropy at sensor location 12. Measuring at this location eliminates 4 scenarios. Thus there are 103 candidate scenarios after measuring at locations 19 and 12. However, at this step, the entropies among model predictions at all potential measurement locations are small. Measuring at any of these locations is unlikely to further reduce the set of candidate damage scenarios. Further improvement is possible by including new sensor types in the sensor placement strategy.

At this stage, engineers may use data mining methodologies, which have been presented in another paper [33], to analyze the set of damage scenarios. Data mining can reveal the number of model classes [34] and determine the most important parameters among the candidate models . An example of the type of results that may be obtained using 
data mining is given in Figure 9. Data mining techniques are used for the visualization of a complex multi-dimensional space of candidate models. In Figure 9, candidate models involving five parameters are presented in terms of their values for only two parameters $\left(\mathrm{p}_{1}, \mathrm{p}_{2}\right)$. Data mining techniques are applied in two steps. First, parameter values are transformed using principal component analysis and next, $\mathrm{K}$-means clustering is employed to find clusters in the transformed data. Figure 9 shows three clusters that are obtained after data mining. Only the first two principal components are plotted in the figure. Representative models from the clusters can provide useful support for engineers during inspection and repair. The potential of such techniques to improve model filtering is currently under study.
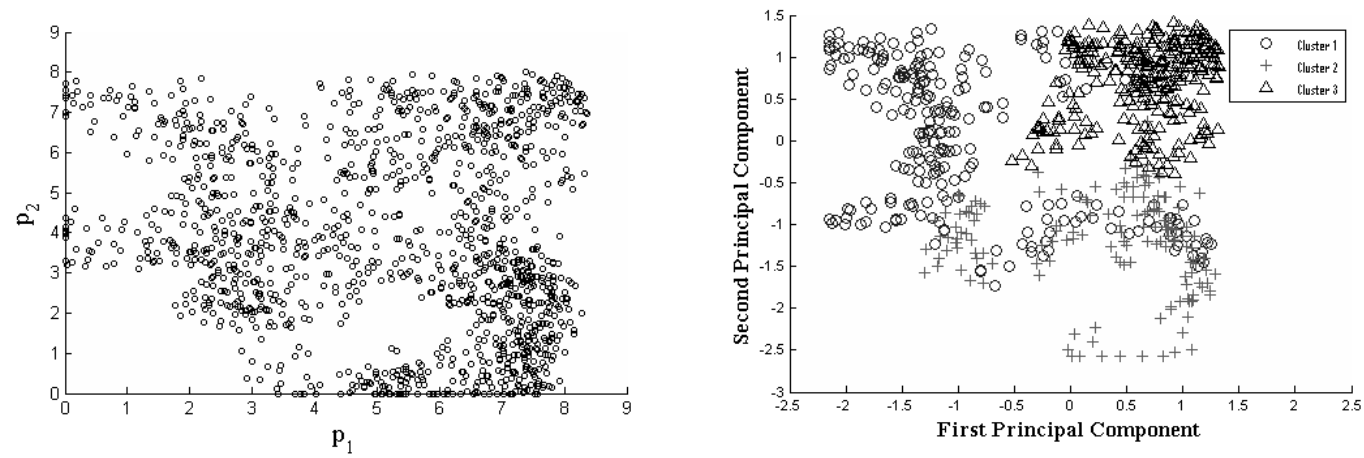

Figure 9: Application of data mining techniques to system identification. On the left is a 2-D projection of a five-dimensional space of candidate models. On the right, three clusters identified by data mining are shown.

\section{Conclusions}

The following conclusions are drawn from this research.

- Global search can be used for designing initial measurement systems that maximize the likelihood of identifying damage scenarios. 
- The maximum number of non-identifiable models gives a good estimate of the performance of a sensor configuration. This is a metric to compare different sensor configurations and choose an optimal number of sensors.

- An entropy-based greedy strategy for sensor placement provides a systematic iterative methodology for detecting damage using the least amount of sensors.

- If the entropies among model predictions are small at all possible sensor locations, then measuring at these locations will not provide additional information. Determining whether or not to install a new sensor is supported using a greedy strategy.

Future work involves experimental error quantification using full scale studies. Experiments in controlled environments are required to estimate probability density functions for measurement and modelling uncertainties. Other important tasks in multiple-model system identification include extending multiple model approach for dynamic measurements and improving engineer-computer interaction for better information visualization.

\section{Acknowledgements}

This research is funded by the Swiss National Science Foundation under contract no. 200020-109257. The authors would like to thank E. Bruhwiler, A. Nussbaumer, B. Raphael, S. Ravindran and S. Saitta for their contributions.

\section{References}

Ni YQ, Zhou HF, Chan KC, Ko JM. Modal Flexibility Analysis of Cable-Stayed Ting Kau

Bridge for Damage Identification. Computer-Aided Civil and Infrastructure Engineering. 2008;23(3):22336. 
[2] Zhang Q, Zhou Y. Investigation of the applicability of current bridge health monitoring technology. Structure and Infrastructure Engineering. 2007;3(2):159 - 68.

[3] Ljung L. System Identification - Theory for the User: Prentice Hall 1999.

[4] Doebling SW, Farrar CR, Prime MB. A Summary Review of Vibration-Based Damage Identification Methods. The Shock and Vibration Digest. 1998 March 1, 1998;30(2):91-105.

[5] Friswell M, Motterhead J. Finite Element Model Updating in Structural Dynamics: Kluwer Academic Publishers 1995.

[6] Sanayei M, Bell ES, Javdekar CN, Edelmann JL, Slavsky E. Damage Localization and FiniteElement Model Updating Using Multiresponse NDT Data. Journal of Bridge Engineering. 2006;11(6):688-98.

[7] Shenton HW, Hu X. Damage Identification Based on Dead Load Redistribution: Methodology. Journal of Structural Engineering. 2006;132(8):1254-63.

[8] Hjelmstad KD, Shin S. Damage Detection and Assessment of Structures from Static Response. Journal of Engineering Mechanics. 1997;123(6):568-76.

[9] Banan MR, Banan MR, Hjelmstad KD. Parameter Estimation of Structures from Static Response. II: Numerical Simulation Studies. Journal of Structural Engineering. 1994;120(11):3259-83.

[10] Ching J, Muto M, Beck JL. Structural Model Updating and Health Monitoring with Incomplete Modal Data Using Gibbs Sampler. Computer-Aided Civil and Infrastructure Engineering. 2006;21(4):242-57.

[11] Jaishi B, Ren W-X. Damage detection by finite element model updating using modal flexibility residual. Journal of Sound and Vibration. 2006;290(1-2):369-87.

[12] Xia P-Q, Brownjohn JMW. Bridge Structural Condition Assessment Using Systematically Validated Finite-Element Model. Journal of Bridge Engineering. 2004;9(5):418-23.

[13] Koh CG, Chen YF, Liaw CY. A hybrid computational strategy for identification of structural parameters. Computers \& Structures. 2003;81(2):107-17.

[14] Sanayei M, Imbaro GR, McClain JAS, Brown LC. Structural Model Updating Using Experimental Static Measurements. Journal of Structural Engineering. 1997;123(6):792-8. 
[15] Fong JT, Filliben JJ, deWit R, Fields RJ, Bernstein B, Marcal PV. Uncertainty in Finite Element Modeling and Failure Analysis: A Metrology-Based Approach. Journal of Pressure Vessel Technology. 2006;128(1):140-7.

[16] Sanayei M, Arya B, Santini EM, Wadia-Fascetti S. Significance of Modeling Error in Structural Parameter Estimation. Computer-Aided Civil and Infrastructure Engineering. 2001;16(1):12-27.

[17] Shenton HW, Hu X. Damage Identification Based on Dead Load Redistribution: Effect of Measurement Error. Journal of Structural Engineering. 2006;132(8):1264-73.

[18] Kripakaran P, Ravindran S, Saitta S, Smith IFC. Measurement System Design Using Damage Scenarios. Computing in Civil Engineering 2007. 40937 ed. Pittsburgh, Pennsylvania, USA: ASCE 2007:73-.

[19] Robert-Nicoud Y, Raphael B, Smith IFC. System Identification through Model Composition and Stochastic Search. Journal of Computing in Civil Engineering. 2005;19(3):239-47.

[20] Liu W, Gao W-c, Sun Y, Xu M-j. Optimal sensor placement for spatial lattice structure based on genetic algorithms. Journal of Sound and Vibration. 2008;317(1-2):175-89.

[21] Meo M, Zumpano G. On the optimal sensor placement techniques for a bridge structure. Engineering Structures. 2005;27(10):1488-97.

[22] Kang F, Li J-j, Xu Q. Virus coevolution partheno-genetic algorithms for optimal sensor placement. Advanced Engineering Informatics. 2008;22(3):362-70.

[23] Papadimitriou C. Optimal sensor placement methodology for parametric identification of structural systems. Journal of Sound and Vibration. 2004;278(4-5):923-47.

[24] Robert-Nicoud Y, Raphael B, Smith IFC. Configuration of measurement systems using Shannon's entropy function. Computers \& Structures. 2005;83(8-9):599-612.

[25] Robert-Nicoud Y, Raphael B, Burdet O, Smith IFC. Model Identification of Bridges Using Measurement Data. Computer-Aided Civil and Infrastructure Engineering. 2005;20(2):118-31.

[26] Garrett JH, Akinci B, Matthews S, Gordon C, Wang HJ, Singhvi V. Sensor data driven proactive management of infrastructure systems. Intelligent Computing in Engineering and Architecture 2006:26284.

[27] Raphael B, Smith IFC. A direct stochastic algorithm for global search. Applied Mathematics and Computation. 2003;146:729-58. 
[28] Domer B, Raphael B, Shea K, Smith IFC. A Study of Two Stochastic Search Methods for Structural Control. Journal of Computing in Civil Engineering. 2003;17(3):132-41.

[29] Shannon C, Weaver W. The Mathematical Theory of Communication: University of Illinois Press 1949.

[30] Saitta S, Raphael B, Smith IFC. Rational design of measurement systems using information science. IABSE Conference. Budapest 2006:118-9.

[31] Billington D. Robert Maillart's Bridges: Princeton University Press 1979.

[32] Maeck J, Peeters B, De Roeck G. Damage Identification on the Z24 bridge using vibration monitoring. Smart Materials and Structures. 2001;10:512-7.

[33] Saitta S, Raphael B, Smith IFC. Data mining techniques for improving the reliability of system identification. Advanced Engineering Informatics. 2005;19(4):289-98.

[34] Saitta S, Kripakaran P, Raphael B, Smith IFC. Improving System Identification Using Clustering. Journal of Computing in Civil Engineering. 2008;22(5):292-302. 


\section{List of figures}

Figure 1: Flowchart of tasks in multiple-model system identification

Figure 2: The residual between model predictions and measurements is plotted for a given parameter value. Each circle is the prediction of a model. The threshold value $(\tau)$ is shown by a dashed line.

Figure 3: Histogram of model predictions

Figure 4: A truss inspired from the railway bridge in Zangenberg, Germany Figure 5: Plot showing the variation of maximum number of non-identifiable models with number of sensors for the bridge in Figure 4

Figure 6: Plan and elevation of Schwandbach bridge in Switzerland

Figure 7: Potential sensor locations on Schwandbach bridge

Figure 8: The variation using global search of maximum number of non-identifiable models with number of sensors for the Schwandbach bridge, and model filtering using a greedy strategy when starting with measurements from an initial measurement system having 4 sensors (vertical dashed line) are shown.

Figure 9: Application of data mining techniques to system identification. On the left is a 2-D projection of a five-dimensional space of candidate models. On the right, three clusters identified by data mining are shown. 